Original Research Article

\title{
Scratch assay microscopy: A reaction-diffusion equation approach for common instruments and data
}

\author{
Alessio Gnerucci ${ }^{\mathrm{a}, *, 1}$, Paola Faraoni ${ }^{\mathrm{b}, 1}$, Elettra Sereni ${ }^{\mathrm{b}, 1}$, Francesco Ranaldi ${ }^{\mathrm{b}}$ \\ ${ }^{a}$ Department of Physics and Astronomy, University of Florence, Via Sansone, 1, 50019, Sesto Fiorentino, Florence, Italy \\ ${ }^{\mathrm{b}}$ Department of Experimental and Clinic Biomedical Sciences "Mario Serio", University of Florence, Viale G. Pieraccini, 6, 50139, Florence, Italy
}

\section{A R T I C L E I N F O}

\section{Keywords:}

Scratch assay

Microscopy

Cell migration

Cell proliferation

Reaction-diffusion equation

\begin{abstract}
A B S T R A C T
Scratch assay is an easy and widely used "in vitro" technique to study cell migration and proliferation. In this work we focus on its modelling and on the capability to distinguish between these two phenomena that the simpler and common models are not able to disentangle.

We adapted a model based on reaction-diffusion equation for being used with common microscopy instruments/data and therefore taking place in the gap between simpler modelling approaches and complex ones. An optimized image analysis pipeline and numerical least-squares fit provide estimates of the scratch proliferation and diffusion coefficients $l$ and $D$.

This work is intended as a first of a series in which the model is tested and its robustness and reproducibility are evaluated. Test samples were NIH3T3 cells scratch assays with proliferation and migration stimulated by varying the foetal bovine serum amount in the culture medium (10\%, 7.5\%, 5\% and $2.5 \%)$. Results demonstrate, notwithstanding an expected $l-D$ anticorrelation, the model capability to disentangle them. The $7.5 \%$ serum treatment can be identified as the model sensitivity limit. Treat-control $l$ and $D$ variations showed an intra-experiment reproducibility $\left(\sim \pm 0.05 / \mathrm{h}\right.$ and $\sim \pm 200 \mu \mathrm{m}^{2} / \mathrm{h}$ respectively) consistent with single fit typical uncertainties $\left(\sim \pm 0.02 / \mathrm{h}\right.$ and $\sim \pm 300 \mu \mathrm{m}^{2} / \mathrm{h}$ respectively).
\end{abstract}

\section{Introduction}

Scratch assay is a simple and widely used "in vitro" technique to study the proliferation and migration in two-dimensional cell cultures. It is based on the observation that after the creation of an artificial gap (called scratch) on a confluent cell monolayer the cells on the edge of the gap will proliferate and move towards the opening progressively closing the scratch until the monolayer is reformed. This "in vitro" technique mimic to some extent migration and proliferation of cells "in vivo" and has become an easy and well-developed tool to study the behaviour of different cellular lines in many physiological or pathological contexts as for example cancer metastasis, embryonic morphogenesis [1], tissue development, immune response or wound healing [2]. It can be ultimately considered as a sensor for a great variety of conditions or chemical/physical stimuli that influence cell migration and proliferation.

Its basic protocol consists in obtaining a confluent monolayer cell culture, creating a scratch on the monolayer and then imaging the scratch at regular time intervals. By means of image analysis and mathematical models the time evolution of scratch closure is studied to extract information about migration and proliferation rates.
An important point is that cell proliferation and migration together cause the progressive closure of the scratch and in many cases it is of central importance to and evaluate their individual contributions. A common method to do this is based on producing samples with proliferation controlled or inhibited with specific drugs or with starvation (a simple protocol consisting in strongly reducing the foetal bovine serum amount in the culture medium [3]). A drawback of this approach is that a confluent monolayer cell culture is itself in an "extreme" condition (i.e. the stationary phase of the growth curve) and treatments such as starvation pull the cells in an even more extreme condition.

In this work we focus instead on the modelling of scratch assay microscopy data and in particular on the possibility to evaluate and disentangle the proliferation and migration contributions by means of a model based on the reaction-diffusion equation.

Moreover our intent is to simplify the experimental approach, both from the modelling than the instrumental point of view, and to make use of common and less demanding instruments/data (i.e. brightfield or phase-contrast low magnification microscopy). Such reactiondiffusion equation based model, that is a commonly used but complex and refined data analysis approach, is therefore adapted and optimized with the above-mentioned simplifying purposes and also dealing with

\footnotetext{
* Corresponding author.

E-mail address: alessio.gnerucci@unifi.it (A. Gnerucci).

1 Authors equally contributed to the realization of this work.
} 
the accuracy and reproducibility limits of the simplest and cheapest data.

In the authors experience with scratch assay literature the more complex and refined models are rare and make use of microscopy techniques complex and less common (i.e. time-lapse microscopy). On the contrary the more common and simple data are studied with too simple models that do not allow distinguishing between proliferation and migration effects that contribute to the scratch closure. The proposed approach aims to be a mid-level complexity one that takes place in the gap between simpler and complex ones and that can give more detailed description of the data also with common and simple microscopy setups. In fact, in many research programmes regarding for example tumour growth or wound healing this assay is yet performed in its basic form (that consist in a few bright-field images of the samples from which the scratch closure rate is estimated) as a side test to support other experiments.

Being this work purpose presenting and discussing a modelling approach we performed experiments aimed at testing the model and evaluating its robustness and reproducibility as a first important step before its adoption. We thus produced samples characterized by slightly different proliferation and migration conditions and then observed how the model interpret and differentiate the various samples.

The cellular model chosen is the common NIH3T3 line and we varied by little amounts the standard foetal bovine serum amount present in the culture medium to obtain samples with slightly different proliferation and migration conditions.

\subsection{Modelling a scratch assay}

The basic principle of the analysis and modelling of a scratch assay is to answer to the question if the closure of a scratch is changed by a given treatment or experimental condition. In practice this is equivalent to answer to the question of which between two scratches closes first.

The basic measurement commonly performed consists in imaging the scratch many times following the time evolution of its closure from the so-called 0 -time to an adequate final time [4,5]. From this set of images a typical geometrical feature of the scratch is measured for each image and its time-evolution is modelled to obtain parameters that characterize and distinguish various samples in a quantitative way.

The most common scratch feature measured is the scratch width, which is the average distance between scratch edges perpendicular to the scratch direction (see for example [3]). Alternatively the surface empty from cells of the scratch is measured $[5,6]$. This quantity is more useful for wound assays spatially limited like spot or hole scratches. Anyway the two geometrical measurements are obviously linked.

Another approach to the scratch assay study is the time-lapse microscopy. In this case the sample is preserved live on the microscope inside a conditioned chamber. Whereas in standard scratch assays the sample is imaged with frequencies from one frame per day to one per hour and immediately after put back in the incubator this approach allows to observe a fixed field of view and follow its changing at frequencies up to some frames per second obtaining a "movie" of the experiment. Time-lapse rather than focusing on the time evolution of global geometrical features of the scratch focuses on following single cells motions and then studying their tracks statistical properties. The advantages of the time-lapse approach are many, but it requires complex instrumentation (i.e. a chamber that encloses part or the entire microscope with controlled temperature, humidity and $\mathrm{CO}_{2}$ concentration).

Coming back to the standard scratch assay microscopy a commonly used parameter to model the scratch closure is the closure-time of the scratch that is the delay time at which the scratch becomes completely closed. This parameter is the one that more directly answers the question "which scratch closes first?" however it is difficult to determine the instant at which the scratch closes completely because this condition varies locally along the scratch and the late scratch evolution, as common for biological and biochemical phenomena, has often an asymptotic behaviour.

Alternatively can be estimated the percentage scratch closure at a fixed delay time that is the percentage ratio between the initial scratch width or area and its value at, for example, $18 \mathrm{~h}$ or $24 \mathrm{~h}$ [7]. Thus, for example, if a sample has a scratch closed to $50 \%$ at $18 \mathrm{~h}$ it means that the scratch at that delay time has half the initial width or empty surface. This parameter can be more easily and robustly estimated with respect to closure time, however it suffers of a strong correlation with the initial scratch width. In fact the same percentage closure correspond to different physical scratch width if the initial width is different, and this can alter the assay interpretation from the biological and biochemical point of view. This fact lead to the important aspect of the experimental reproducibility of the initial scratch width and thus of the method adopted for producing a scratch on a cell monolayer. In the experiments performed for this work we produced scratches ranging from $\sim 600 \mu \mathrm{m}$ to $\sim 1400 \mu \mathrm{m}$ with a reproducibility of $\sim 200 \mu \mathrm{m}$ (many samples were realized and only the less variable triplicate was selected for the experiment continuation). Therefore this problem cannot be ignored, and a robust analysis method has to take into account this aspect.

Another approach consists in modelling the time evolution of the scratch width with a linear model and estimating its average closure velocity (i.e. the angular coefficient of the best-fit linear model). The advantage of studying this parameter is that it is not correlated to the initial scratch width.

Alternatively a common the scratch width vs. time curve is modelled with a sigmoid (or logistic) curve [8,9]. This model is characterized by 3 parameters and thus it gives more information on the scratch closure. However robustly constraining it requires more data points than the linear model. The choice of which of these two models adopt is principally influenced by the quality of the data obtained.

As previously observed all these basic models are not able to distinguish between cell proliferation and migration contributes to the scratch closure. The strategy chosen in this work consist therefore, as a first step, in modelling the time evolution of the scratch with a linear model: the basic yet more common model adopted. The results of this model provide a reference and are integrated with a more complex modelling, the principal subject of the work, able to distinguish between cell proliferation and migration. As observed above this complex model is adapted and optimized, with a simplifying purpose, for being used with the common microscopy instruments/data also used for the basic models.

The model is based on the reaction-diffusion equation (or Fisher equation, hereafter RDE [10-15]) that allows to calculate the timeevolution of the cell density profile perpendicular to the scratch. This model depends on two main parameters: the diffusion coefficient that represents the sample migration attitude and the proliferation coefficient. Therefore it allows the above mentioned identification and disentanglement of the contribution to scratch closure of the two phenomena. As previously observed, a more complex model requires better data, therefore our approach is to consider both the basic and the complex ones and not neglect the former in favour of latter. Moreover, different models give different information on the same experiment and thus they have to be considered as complementary.

Some works in literature indicates that RDE, despite is a common and frequently used model, is not the more accurate one to describe the phenomenon of collective cell migration and proliferation $[15,16]$. The process of developing and optimizing a modelling approach needs to take into account the aspect of the model choice. However the principal aim of this work is to optimize a particular model for being used with data never modelled with it: the simplest and cheapest brightfield images. In the context of the development of a complete modelling procedure, and especially of the image analysis steps associated and preparatory to it we preferred adopting the model in its basic form: RDE. 


\section{Material and methods}

\subsection{Cell culture - scratch assay}

NIH3T3 cells were seeded $\left(2.0 \cdot 10^{5}\right)$ on $60 \mathrm{~mm}$ collagen-coated Petri dishes and cultured in Dulbecco's modified Eagle medium (DMEM, SIGMA, Italy) containing $4500 \mathrm{mg} / \mathrm{L}$ glucose and $2 \mathrm{mM}$ L-glutamine supplemented with $10 \%$ foetal bovine serum until $~ 80 \%$ of confluence. Then monolayers were gently scratched with a $200 \mu 1$ pipette tip. Culture medium and detached cells were removed and monolayers were washed twice with phosphate buffer solution (PBS). Fresh complete medium was added for control samples or substituted with DMEM at different foetal bovine serum concentration $(7.5 \%, 5 \%$ and $2.5 \%)$. Samples where then observed at the microscope as detailed in the following subsection: this moment is considered 0 delay time of the scratch closure to which we refer in the following.

Experiments were performed realizing twin scratch assays with the role of control and treat. Each control-treat experiment was performed in triplicate.

\subsection{Microscopy}

Scratch assay imaging was performed with an inverted microscope in phase contrast configuration (Leica DM IL, Leica Microsystems Wetzlar $\mathrm{GmbH}$ ) equipped with a $5 \mathrm{x}$ objective and a Canon CCD camera (Canon Power shot S40, $2272 \times 1074, \sim 23 \times 20 \mu \mathrm{m}$ pixels, Canon Inc. Japan).

The $5 \mathrm{x}$ objective was chosen as an adequate magnification to obtain a field of view of $\sim 2.5 \times 2.5 \mathrm{~mm}$ necessary to observe the entire scratch width $(\sim 0.6-1.4 \mathrm{~mm})$. By imaging a Burker chamber and measuring the distance between grooves that is known from chamber specifications was measured the image scale in $\mu \mathrm{m} / \mathrm{pix}$.

Images of the scratch were acquired approximately once an hour from the starting time (hereafter t0) to $\sim 25-40 \mathrm{~h}$ (time when typically the scratch was almost closed). We choose a high image acquisition rate compared to what present in literature (down to 1-2 images per day) and to the typical scratch closure velocity. Our intent was to have curves well sampled to take into account also of the uncertainties of the scratch image measurements and to allow a good least squares fit.

Obviously the time sampling of our experiments suffered of a unavoidable night-time gap because them lasts $25-40 \mathrm{~h}$ and no automatization was available. However the $1 \mathrm{~h}$ sampling guaranteed curves with $10-15$ points.

\subsection{Image analysis and modelling}

Image analysis was performed by means of ImageJ Fiji software (http://fiji.sc/Fiji).

Scratch assay modelling was performed by means of ImageJ Fiji software, Excel datasheet (https://www.microsoft.com) and dedicated Python routines (https://www.python.org/).

Single images were acquired in Jpg format selecting for the analysis the red channel that have the higher contrast. Images were rebinned with a $3 \times 3$ pixel window and a contrast equalization filter was applied to increase the image signal to noise ratio and contrast (Fig. 1, panel A). The final estimated spatial scale was $6.226 \mu \mathrm{m} /$ pix. A variance filter with 2 pix square window was applied and then a threshold filter of the resulting image allowed identifying the region of interest (ROI) corresponding to the empty scratch surface (Fig. 1, panel B to D). Measuring the surface of the scratch-ROI and dividing it for the image height allowed to calculate the scratch width (hereafter Sw). Obviously this operation required that scratch be aligned to the vertical image axis. An error in performing this alignment of a $\sim 15^{\circ}$ angle (i.e. an estimate of the maximum error that an operator can introduce judging by eye) results in an overestimate of $\mathrm{Sw}$ of $\sim 3.5 \%$. This indicates that a bad alignment can introduce uncertainties not higher than other uncertainty sources.

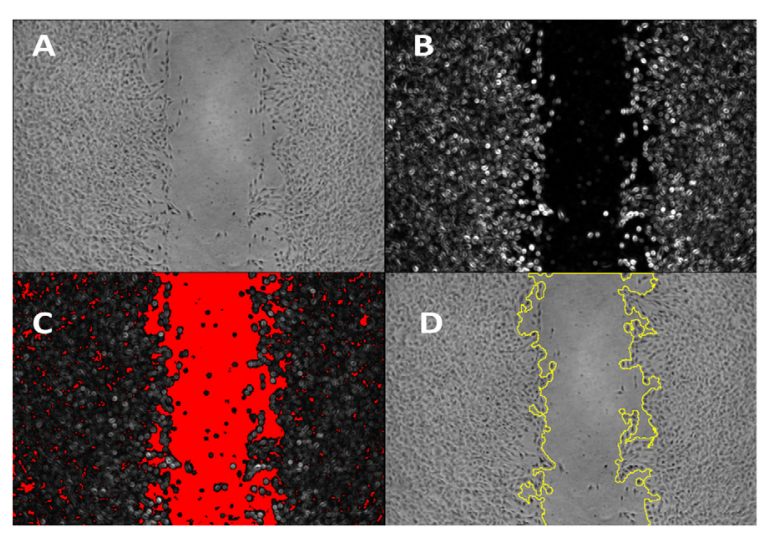

Fig. 1. Example of the image analysis. Panel A: original image (red channel) rebinned and contrast enhanced. Panel B: variance filter. Panel C: threshold filter. Panel D: scratch ROI identification.

\subsubsection{Linear model}

Image analysis allows obtaining the scratch-width vs. delay time curve. This curve is modelled by means of least squares minimization with a linear model:

$S_{w}(t)=S_{w 0}-v t$

where $S_{w 0}$ is the initial scratch width and v the average closure velocity (i.e. the slope of the straight line model). We can observe that this model allows also to calculate the closure-time of the scratch $T_{c}$ that is the time at which the linear model intercept the $\mathrm{x}$ axis:

$T_{c}=S_{w 0} / v$

\subsubsection{Sigmoid model}

This model has the following functional form:

$S_{w}(t)=\frac{A}{1+e^{\left(4 v_{\max }\left(t-t_{\max }\right) / A\right)}}$

where $A$ is a scale amplitude, $v_{\max }$ the maximum closure velocity and $t_{\max }$ the time at which the scratch closes at its maximum velocity. In this work we performed also the sigmoid modelling but decided to not discuss the results because the number of curve points and the unavoidable night time gap in data did not allow a reliable least squares fit.

\subsubsection{Reaction-diffusion equation model}

One-dimensional RDE ha the following form:

$\frac{\partial P(x, t)}{\partial t}=D \frac{\partial^{2} P(x, t)}{\partial x^{2}}+l P(x, t)\left(1-\frac{P(x, t)}{K}\right)$

where $P(x, t)$ is the one-dimensional cell density profile (function of the $x$ coordinate perpendicular to the scratch and of time), $D$ is the diffusion coefficient, $l$ is the proliferation coefficient and $K$ the carrying capacity of the system (i.e. the limit density of the cell monolayer). While the first member of equation describes the diffusion process (i.e. the cell migration) the second member describe the logistic cell growth curve component.

$D$ represents the cell migration attitude and it makes $P(x, t)$ evolve the scratch shrinking and its edges becoming softer because cells moves from high density regions to low density ones. $l$ instead represents the cell proliferation attitude and makes $P(x, t)$ evolve the scratch shrinking but its edges remaining steep because low density regions are filled by the cell number growth. Therefore if scratch profile evolves with steeper edges this indicate that proliferation dominates whereas if them evolve with a softer shape migration dominates.

Whereas the linear model calculates the scratch width as a function of time $\left(S_{w}(t)\right)$ RDE model calculates a quantity that is both a function 
of space and time $(P(x, t))$. Therefore the former approach is based on measuring and modelling a single value at each time ( $S_{w}$ "at each $t$ ") and the latter on measuring and modelling a function of the position at each time $(P(x)$ "at each $t$ "). For this reason is not possible a direct comparison of the two models results. Our strategy will be to use the linear model to estimate the mean scratch closure velocity $v$ on the various samples and then the RDE model to interpret and enrich these results by estimating the diffusion and proliferation coefficients.

The first step is the measurement of $P(x, t)$ starting from the images acquired at each delay time. In [15] $P(x, t)$ is measured by simply counting the cells in the image. In detail scratch is aligned to image $y$-axis then image is divided in small vertical slices each associated with its mean $x$ coordinate and for each slice cells are counted and density is calculated dividing by the slice surface. Such a profile is obtained from a 2D image with an average along the $y$-axis and is in fact one-dimensional (function of $x$ coordinate). In this work we developed a different measure of $P(x, t)$. An adaptive threshold filter was applied to each image. This filter is one of the simplest yet useful cell segmentation tools that produces a binary image that identifies quite well single cell as disconnected ROIs at which is attributed a pixel value of " 1 " and the background ROIs at which is attributed the value " 0 " (see Fig. 2). Each binary image is then averaged along its $y$-axis to obtain a one-dimensional profile function of $\mathrm{x}$ that we call $P^{m}(x, t)$ :

$P^{m}(x, t)=\frac{N_{p i x}^{\text {Cell }}(x, t) \cdot 1+N_{p i x}^{\text {Back }}(x, t) \cdot 0}{N_{p i x}^{\text {Tot }}(x, t)}=\frac{N_{p i x}^{\text {Cell }}(x, t)}{N_{p i x}^{T o t}(x, t)}$

where $N_{\text {pix }}^{\text {Cell }}(x, t), N_{\text {pix }}^{\text {Back }}(x, t)$ and $N_{p i x}^{T o t}(x, t)$ are respectively the pixel number owning to the cell ROIs, background ROIs and the total pixel number of the column at $x$ coordinate. If we observe that

$N_{\text {pix }}^{\text {Cell }}(x, t)=\bar{n}_{\text {pix }}^{\text {Cell }} \cdot N^{\text {Cell }}(x, t)$,

where $\bar{n}_{p i x}^{C e l l}$ is the average pixel number owning to a single cell and $N^{C e l l}(x, t)$ is the number of cells, we obtain:

$P^{m}(x, t)=\frac{\bar{n}_{p i x}^{\text {Cell }} \cdot N^{\text {Cell }}(x), t}{N_{p i x}^{\text {Tot }}(x, t)}=\bar{n}_{p i x}^{\text {Cell }} \cdot \Sigma^{\text {Cell }}(x, t)$

where $\Sigma^{C e l l}(x, t)$ is the needed cell number-density as a function of $x$ and $t$. In the approximation that $\bar{n}_{p i x}^{\text {Cell }}$ does not vary too much with the $x$ coordinate, the quantity $P^{m}(x, t)$ is effectively proportional to the cell density profile and can be used in its place differing only for a scale factor. The average operation along the $y$-axis, and thus over more cells at that $x$ coordinate make this approximation robust. This approach to measure $P^{m}(x, t)$ distinguish our modelling procedure from other works: in [15], for example, an incubating time-lapse cell imaging facility is used and the same profile is obtained by means of single cell counting. Our approach, on the contrary, is faster and simpler coherently with the simpler nature of imaging data and their possible worse accuracies.

To take into account that $P^{m}(x, t)$ is proportional to cell number density by an unknown scale factor we chose to normalize them to a constant value " 1 " outside the scratch (i.e. on the monolayer, see Fig. 2 panel C), the RDE fit is then performed in adimensional units and subsequently rescaled to cell number-density as explained in the following. Normalization is made fitting a 2nd degree polynomial function only on the monolayer region and then dividing the profile by this function. This operation allows also taking into account the possible uneven microscope illumination in the images.

To avoid spatial oversampling the profiles $P^{m}(x, t)$ are rebinned with a 6 bin window for a final scale of $36 \mu \mathrm{m} /$ bin (a bin dimension adequate to the typical spatial-scale of the cells that are the "unit" agents of the model). Moreover, the profiles are spatially limited to a few bins outside the scratch $(\sim 100 \mu \mathrm{m}$ or $3-4$ points) to avoid underestimating the statistical weight of the points owning to the scratch edges that, as previously observed, are the most important for the model.

$\mathrm{RDE}$ is not solvable analytically and the fit is performed with a numerical solution of the equation calculated on a grid of $x$ and $t$
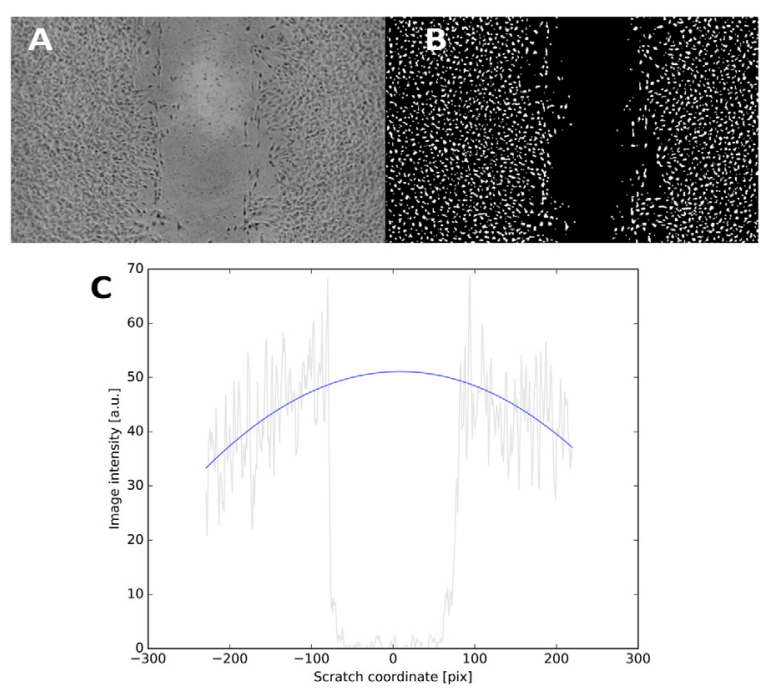

Fig. 2. Example of the image analysis pipeline. Panel A: original image (red channel) rebinned and contrast enhanced. Panel B: binary image from the adaptive threshold filter. Panel C: one-dimensional scratch profile from the average of binary image along the y axis (thin black line, arbitrary units). Continuous blue line represents the polynomial fit of the profile outside the scratch used for the normalization of the profile.

values $\left(x_{g}, t_{g}\right)$. This solution is valid if the grid step $\left(\Delta x_{g}, \Delta t_{g}\right)$ follows the condition: $D \cdot \delta t_{g} \ll \Delta x_{g}^{2}$ (see for example [17]). The equation starts from the t0 profile $P^{m}(x, 0)$ and evolves it step-by-step at subsequent times calculating the model-profiles $P^{M o d}\left(x_{g}, t_{g}\right)$ normalized as the measured profiles. Therefore $P^{m}(x, 0)$ is actually used by the model as an input parameter.

To rescale both the normalized $P^{m}(x, t)$ and $P^{M o d}\left(x_{g}, t_{g}\right)$ cell number-density was measured for a subset of images relative to lagtimes $t^{\prime}$ (hereafter $\Sigma_{M}\left(t^{\prime}\right)$ ). In detail square surfaces located outside the scratch (i.e. on the monolayer) are selected and the cell number-density is measured manually counting the cell inside the square and dividing by its surface (see for example [14]). Being outside and far from the scratch the variation in time of $\Sigma_{M}\left(t^{\prime}\right)$ is only due to proliferation. Therefore it can be modelled with a logistic growth curve (see for example [18])

$\Sigma_{M}\left(t^{\prime}\right)=\frac{K \Sigma_{0}}{\Sigma_{0}-\left(\Sigma_{0}-K\right) e^{-l t^{\prime}}}$

where the various parameters are the ones previously defined and $\Sigma_{0}$ is the starting monolayer cell number-density that is the key parameter to rescale to cell number density the whole model. The least squares fit of $\Sigma_{M}\left(t^{\prime}\right)$ is the first step of the whole procedure.

It is important to observe that to robustly fit this growth curve model it is fundamental to observe a variation of $\Sigma_{M}\left(t^{\prime}\right)$ during the experiment and thus the initial monolayer density $\Sigma_{M}(0)$ has to be adequately lower than its limit value. This is another reason that justifies the choice of a monolayer cell confluence of $\sim 85 \%$ for the scratch assay.

Subsequently the procedure calculates the normalized density profiles $P^{M o d}\left(x_{g}, t_{g}\right)$ and extrapolate them to the measured $(x, t)$ coordinates to fit the measured $P^{m}(x, t)$. Regarding the model parameters $\Sigma_{0}$ is fixed being the global cell-density scale factor, $K$ and $l$ are free to vary, but with a limited range of \pm 5 standard deviations from the $\Sigma_{M}\left(t^{\prime}\right)$ fit results (to allow this first fit to partially constrain the global fit) and $D$ is completely free to vary.

The model considers another parameter: the start-time $T_{S}$ : an initial lag-time before which the sample remains freezed and after which the scratch closure start. This parameter has been introduced to finelytune the fit quality maintaining its interpretation simple. Various values 
A

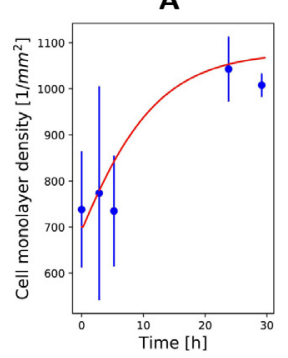

B

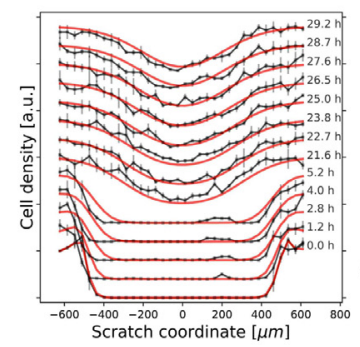

C

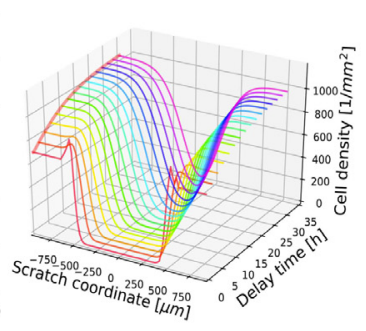

D

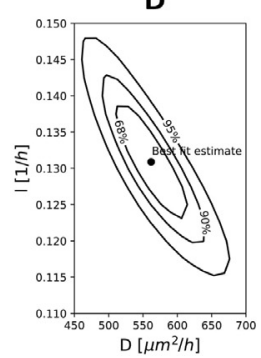

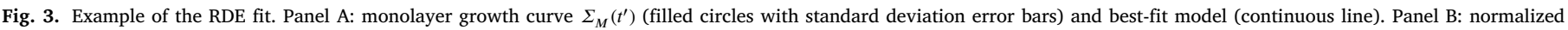

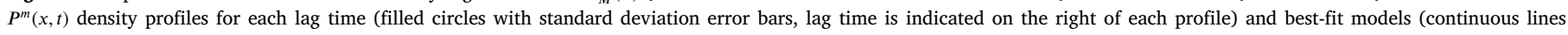

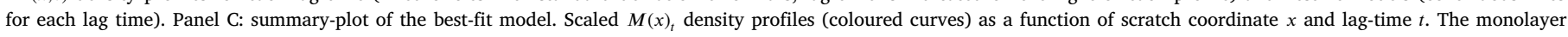

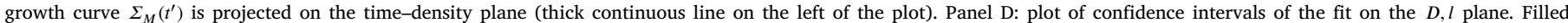

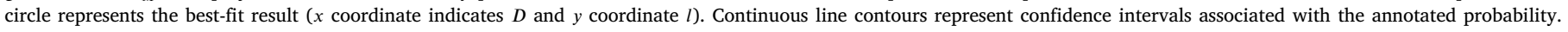
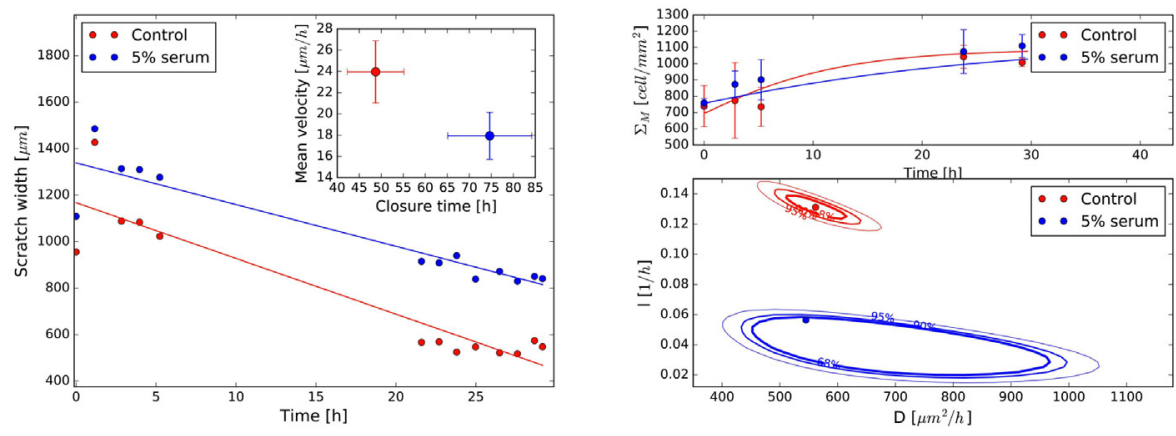

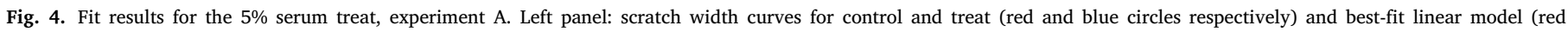

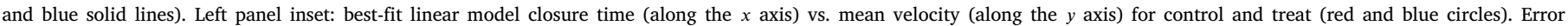

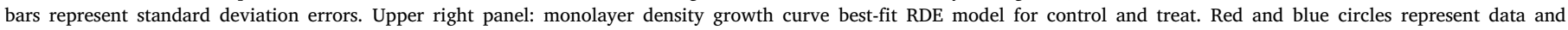

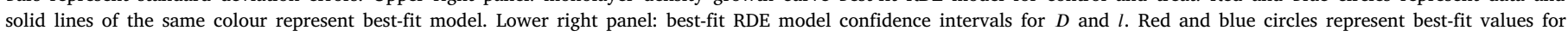
treat and control samples respectively ( $x$ coordinate represents $D$ and y coordinate $l$ ). Contours of the same colour represent the relative confidence intervals.

have been tested and then a fixed value of 15 ' $(0.25 \mathrm{~h})$ has been chosen for all the samples.

In Fig. 3 is shown an example of the fit results: sample monolayer growth curve and relative best-fit model (Fig. 3, panel A); normalized $P^{m}(x, t)$ profiles and their best-fit model (Fig. 3, panel B). In panel C of Fig. 3 is summarized the complete model scaled to cell number-density. In the time-density plane is projected the modelled monolayer growth curve (red thick line).

The most important parameters of this model are the proliferation coefficient $l$ and the diffusion coefficient $D$ that represent the samples proliferation and migration attitude respectively. To determine their uncertainties and correlations the analysis of confidence intervals was performed (panel D of Fig. 3). Confidence intervals are the regions in the $D, l$ plane associated to a given probability to find the bestfit within. For example the $68 \%$ contour define the region for which there is a probability of $68 \%$ that best-fit $D, l$ values fall inside. It can be viewed as a two-dimensional uncertainty region around the bestfit solution that is the point with $x$ and $y$ coordinate corresponding to $D$ and $l$ respectively (panel D of Fig. 3). Moreover the shape of the region indicates the correlation degree of the two model parameters (region contours almost circular indicate low correlation between D and 1 whereas elongated contours indicate a high correlation or anticorrelation). Confidence intervals are calculated following the method described in [19] that is based on repeating the fit fixing the D and 1 values couples on a grid on the $D, l$ plane and for each grid point calculating the fit $\chi^{2}$. These $\chi^{2}$ values can be associated to the probability to obtain that particular results by means of integrals of the $\chi^{2}$ distribution with the proper number of degree of freedom (see again [19] for statistical details).

\section{Results}

\section{1. $5 \%$ serum culture medium}

In Figs. 4 to 6 results of experiments A, B, C for the 5\% serum treatment are presented respectively. In left panel of each figure are resumed the linear model results. In the main plots are shown the scratch width vs. time curves for treat and control samples (filled circles) and the relative best-fit linear models (continuous lines of the same colour). In the left panels insets the best-fit model parameters are plotted with the same colour of the relative data: the mean velocity $v$ (i.e. the slope of the linear model) along the $y$-axis and the closure time $T_{C}$ along the $x$-axis. Error bars represent standard deviation errors. In right panels are resumed the results of the RDE modelling. In the upper right panels are shown the fit of monolayer density growth curves for treat and control samples (filled circles for the data and continuous lines of the same colour for the best-fit model). In lower right panels are shown the confidence intervals for $D$ and $l$ values. Filled circles represent the best-fit results for $D$ and $l$ (along the $x$ and $y$ axis respectively) for treat and control samples. Contours of the same colour represent the confidence intervals for that particular sample.

Comparing the results on $\mathrm{A}, \mathrm{B}, \mathrm{C}$ repetitions of the experiment for each modelled parameter ( $v$ form linear model and $l$ and $D$ from RDE model) it can be observed that the best-fit parameter values are not always well reproduced (i.e. the dispersion of the results is sometimes higher than the typical uncertainty of a single fit). The variation from treat to control of each parameter is instead better reproduced. In the following we will detail and discuss this fact.

Regarding the linear model the best-fit values of $v$ for equivalent samples of A, B, C experiments varies by $\sim 2 \mu \mathrm{m} / \mathrm{h}$ where the typical fit uncertainties are $\sim 1.5 \mu \mathrm{m} / \mathrm{h}$. This indicates that $v$ is well reproduced on the three experiment repetitions. 

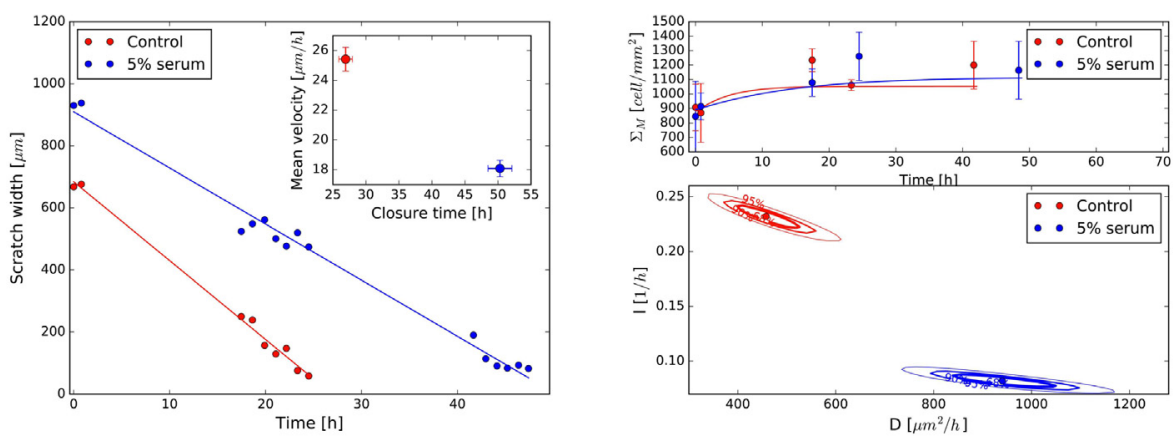

Fig. 5. Fit results for the $5 \%$ serum treat, experiment B. Same indications of the previous figure.
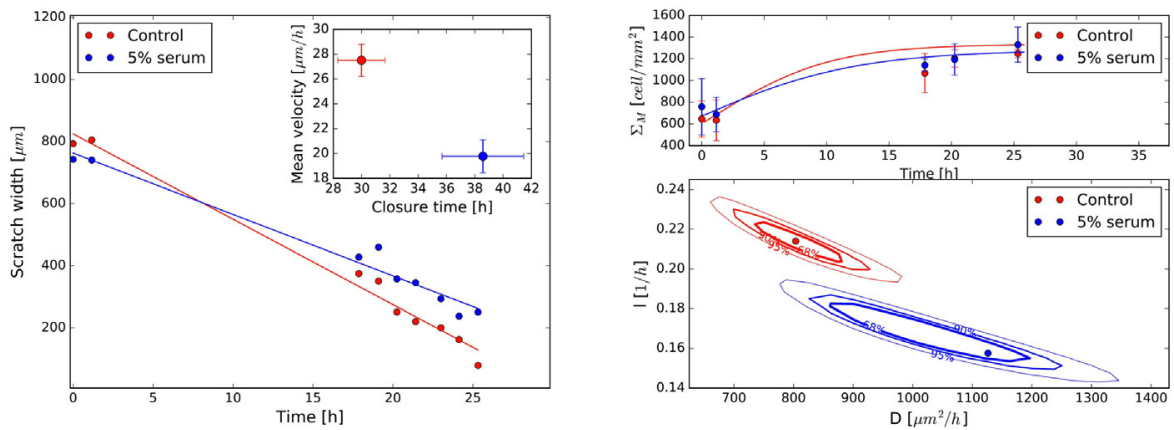

Fig. 6. Fit results for the $5 \%$ serum treat, experiment C. Same indications of the previous figure.

Treat-control variations of $v$ (hereafter $v_{T-C}$ ) are $-6 \mu \mathrm{m} / \mathrm{h},-7 \mu \mathrm{m} / \mathrm{h}$ and $-8 \mu \mathrm{m} / \mathrm{h}$ respectively for experiments $\mathrm{A}, \mathrm{B}$ and $\mathrm{C}$ with an average and standard deviation of $-8 \pm 2 \mu \mathrm{m} / \mathrm{h}$ : this standard deviation of $\pm 2 \mu \mathrm{m} / \mathrm{h}$ can be considered an estimate of the intra-experiment reproducibility of the linear model for $v_{T-C}$. Regarding the couple control-5\% serum can be concluded that the linear model shows a statistically significant decrease of closure velocity for the treated sample.

As previously observed, the situation is different for the RDE model. Best-fit values for equivalent samples of A, B, C experiments are not equally well reproduced by the model: them varies by $\sim 0.1 / \mathrm{h}$ and $\sim 600 \mu \mathrm{m}^{2} / \mathrm{h}$ respectively for $l$ and $D$ : higher than the typical confidence interval extent $\left(\sim 0.02 / \mathrm{h}\right.$ for $l$ and $\sim 300 \mu \mathrm{m}^{2} / \mathrm{h}$ for $\left.D\right)$.

The 1 treat-control variation (hereafter $l_{T-C}$ ) are $-0.1 / \mathrm{h},-0.15 / \mathrm{h}$ and $-0.06 / \mathrm{h}$ respectively for experiments $\mathrm{A}, \mathrm{B}$ and $\mathrm{C}$ with an average and standard deviation of $-0.1 \pm 0.05 / \mathrm{h}$. $D$ shows a treat-control variation (hereafter $D_{T-C}$ ) of $100 \mu \mathrm{m}^{2} / \mathrm{h}, 500 \mu \mathrm{m}^{2} / \mathrm{h}$ and $300 \mu \mathrm{m}^{2} / \mathrm{h}$ respectively for experiments A, B and C with an average of $300 \pm$ $200 \mu \mathrm{m}^{2} / \mathrm{h}$.

The intra-experiment reproducibility of $l_{T-C}$ and $D_{T-C}$ are $\pm 0.05 / \mathrm{h}$ and $\pm 200 \mu \mathrm{m}^{2} / \mathrm{h}$ respectively: consistent or slightly higher than the typical extent of the confidence intervals for the two parameters.

The obtained results show that the RDE model interprets the scratch velocity closure reduction resulting from linear model as a reduction of the proliferation coefficient $l$ (i.e. proliferation attitude) and a smaller increase of diffusion coefficient $D$ (i.e. migration attitude).

\section{2. $7.5 \%$ serum culture medium}

In Figs. 7 to 9 results of experiments A, B, C for the 7.5\% serum treatment are presented respectively. In left panel of each figure are shown the scratch width curves and best-fit linear models (continuous lines). Left panels insets show the relative best-fit mean velocities and closure times. In right panels are shown the RDE model fit of monolayer growth curve (upper right panels) and confidence intervals for the D and 1 values (lower right panels).
Regarding the linear model the best-fit values of $\mathrm{v}$ for A, B, C experiments varies by $\sim 2 \mu \mathrm{m} / \mathrm{h}$ : with typical fit uncertainties of $\sim 1.5 \mu \mathrm{m} / \mathrm{h}$. $v_{T-C}$ results are instead $-4 \mu \mathrm{m} / \mathrm{h},-5 \mu \mathrm{m} / \mathrm{h}$ and $0 \mu \mathrm{m} / \mathrm{h}$ respectively for experiments A, B and C with an average and standard deviation of $-33 \mu \mathrm{m} / \mathrm{h}$ : slightly higher than typical fit uncertainties.

In conclusion for the couple control-7.5\% serum the linear fit does not shows any statistically significant variation.

Regarding RDE model results 1 and D best-fit values for A, B, C experiments vary by $\sim 0.06 / \mathrm{h}$ and $\sim 300 \mathrm{~h} / \mu \mathrm{m}^{2}$ respectively: higher than the typical confidence interval extent for the two parameters $(\sim 0.02 / \mathrm{h}$ for $l$ and $\sim 300 \mu \mathrm{m}^{2} / \mathrm{h}$ for $D$ ). Regarding treat-control variations $l_{T-C}$ fit results are $-0.06 / \mathrm{h},-0.02 / \mathrm{h}$ and $+0.05 / \mathrm{h}$ respectively for experiments $\mathrm{A}, \mathrm{B}$ and $\mathrm{C}$ with an average and standard deviation on the three experiments of $0.0030 .0045 / \mathrm{h} . D_{T-C}$ fit results are $+50 \mu \mathrm{m}^{2} / \mathrm{h},-100 \mu \mathrm{m}^{2} / \mathrm{h}$ and $-100 \mu \mathrm{m}^{2} / \mathrm{h}$ respectively for experiments A, B and C with an average and standard deviation of $-4090 \mu \mathrm{m}^{2} / \mathrm{h}$.

The intra-experiment reproducibility of $l_{T-C}$ and $D_{T-C}$ are $0.0045 / \mathrm{h}$ and $90 \mu \mathrm{m}^{2} / \mathrm{h}$ respectively and result consistent with the typical extent of the confidence intervals along the two parameters axis.

The RDE modelling, taken into account the variability on three experiment repetitions, confirms the results of linear modelling that the $7.5 \%$ serum treat do not show any statistically significant variation with respect to control.

\subsection{Monolayer confluence test}

In Fig. 10 the results of a single experiment for a $2.5 \%$ serum treatment are presented. In this particular test was evaluated the effect on the scratch closure of two different initial monolayer densities maintaining fixed the $2.5 \%$ serum culture medium for both samples.

In left panel is shown the scratch width curve linear model fit (continuous lines). Left panel inset shows the relative best-fit mean velocities and closure times. Right panel shows the RDE model fit of monolayer growth curve (upper right) and confidence intervals for the $D$ and $l$ values (lower right).

Linear model results show a $v_{T-C}$ increase from high density to low density sample of $12 \mu \mathrm{m} / \mathrm{h}$ with typical fit uncertainties of $\sim 1.5 \mathrm{~m} / \mathrm{h}$. 

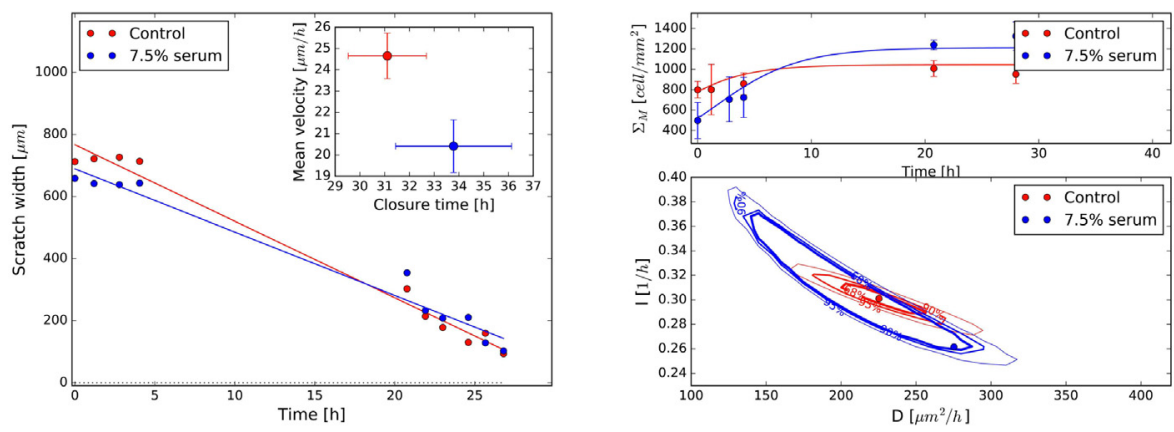

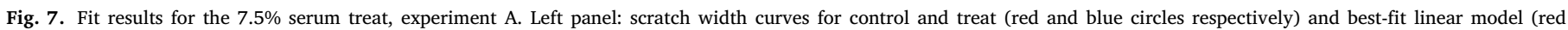

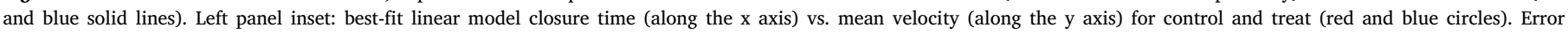

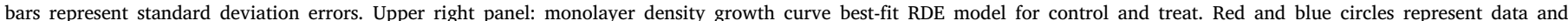

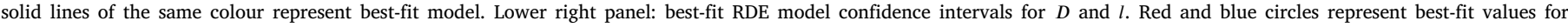
treat and control samples respectively ( $x$ coordinate represents $D$ and $y$ coordinate $l$ ). Contours of the same colour represent the relative confidence intervals.
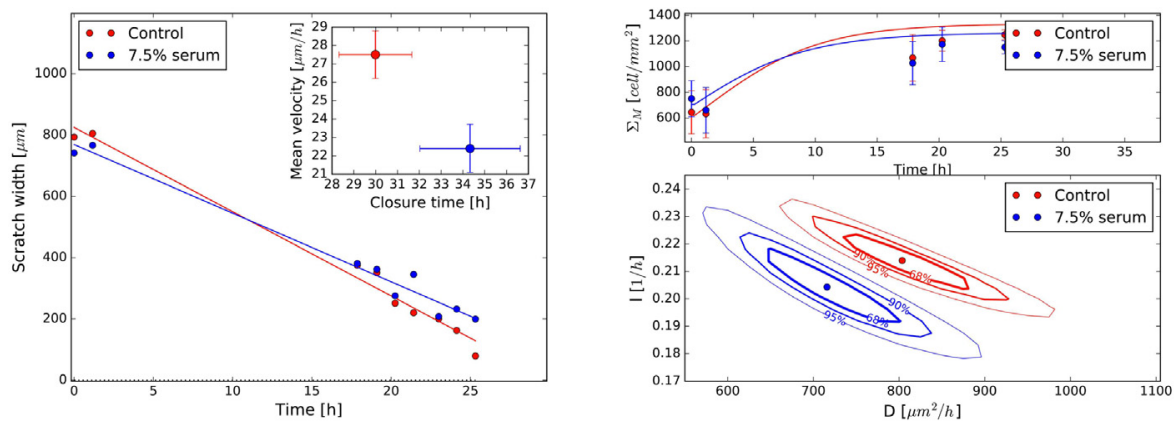

Fig. 8. Fit results for the $7.5 \%$ serum treat, experiment B. Same indications of the previous figure.

RDE model results shows $l_{T-C}$ and $D_{T-C}$ variations from high density to low density of $\sim 0.06 / \mathrm{h}$ and $+500 \mu \mathrm{m}^{2} / \mathrm{h}$ respectively with typical confidence interval extents of $\sim 0.01 \mu \mathrm{m} / \mathrm{h}$ for $l$ and $\sim 100 \mu \mathrm{m}^{2} / \mathrm{h}$ for $D$.

This test shows that halving the initial monolayer density (from $\sim 1000 \mathrm{cell} / \mathrm{mm}^{2}$ of high density sample to $\sim 500 \mathrm{cell} / \mathrm{mm}^{2}$ as shown in upper right panel of Fig. 10) causes a faster scratch closure interpreted by the RDE model as an increase of both the proliferation and the migration attitude of sample.

\section{Discussion}

In this work we presented a modelling approach of the scratch assay microscopy aimed at estimating the proliferation and migration components that determine the scratch closure and tested the robustness and reproducibility of its results with dedicated experiments.

Low magnification images of the scratch samples were acquired through a common inverted microscope in phase contrast configuration for about $25-40 \mathrm{~h}$ at a frequency of $\sim 1 / \mathrm{h}$. We developed an image analysis pipeline to measure in a fast and easy way a one-dimensional average density profile along a direction orthogonal to the scratch and discussed that it is, with a good approximation, proportional to the cell number-density. This data were least squares fitted with a dedicated routine based on the numerical solution of the reactiondiffusion equation (RDE) obtaining thus estimates of the proliferation coefficient $l$ and diffusion coefficient $D$.

The analysis of experiments triplicates allowed comparing the dispersion of the results with the typical single fit uncertainties.

The best-fit $l$ and $D$ values show on the triplicates variations higher than their typical single fit uncertainties (represented by the extent of confidence intervals). For example for the $5 \%$ serum experiment $l$ and $D$ varies of $\sim 1 / \mathrm{h}$ and $\sim 600 \mathrm{~m}^{2} / \mathrm{h}$ with single fit uncertainties of $\sim 0.02 / \mathrm{h}$ and $\sim 300 \mu \mathrm{m}^{2} / \mathrm{h}$. This might indicate bad intra-experiment model reproducibility. However we observed that the treat-control variations of each parameter are better reproduced on the triplicate: for example for the $5 \%$ serum experiment $l_{T-C}$ and $D_{T-C}$ are $0.1 \pm$ $0.05 / \mathrm{h}$ and $300 \pm 200 \mu \mathrm{m}^{2} / \mathrm{h}$. Their standard deviations on the triplicate ( $\sim 0.05 / \mathrm{h}$ and $\sim 200 \mu \mathrm{m}^{2} / \mathrm{h}$ ) are consistent or slightly higher than the typical single fit uncertainties $\left(\sim 0.02 / \mathrm{h}\right.$ and $\left.\sim 300 \mu \mathrm{m}^{2} / \mathrm{h}\right)$ and can be considered as an estimate of the intra-experiment model reproducibility and definitely more robust and prudent estimates of the modelling method uncertainties.

For the $7.5 \%$ serum experiment $l_{T-C}$ and $D_{T-C}$ are $0.003 \pm 0.0045 / \mathrm{h}$ and $40 \pm 90 \mu \mathrm{m}^{2} / \mathrm{h}$, but for this experiment the estimate of intraexperiment reproducibility is less reliable being $l_{T-C}$ and $D_{T-C}$ themselves consistent with 0 .

The calculation of confidence intervals for $l$ and $D$ indicates that the fit is characterized by an inverse correlation between the two parameters. This is consistent with the fact that this model describes the closure of a scratch by means of two competitive phenomena: cell proliferation and migration. The same scratch evolution can be obtained either with a lower migration attitude and a higher proliferation or in an inverse case.

This fact in principle limits the capability of the fit to disentangle $l$ and $D$, but the result obtained for the sample with $5 \%$ serum indicates that, taken into account of both single fit uncertainties and intraexperiment reproducibility, treat can be distinguished from control and this in turn indicates that the model can partially disentangle $l$ from $D$. In particular the scratch closure slowing down due to the $5 \%$ serum treat is interpreted by the RDE model with a reduction of the proliferation coefficient $l$ (an effect probably due to the use of a low-serum culture medium) and an increase of diffusion coefficient $D$.

In the case of the $7.5 \%$ serum instead treat and control are statistically consistent both for $l$ and $D$. This particular treatment can be thus considered as a sensitivity limit of the modelling method: increasing the serum from $5 \%$ to $7.5 \%$ (closer to the $10 \%$ of the 

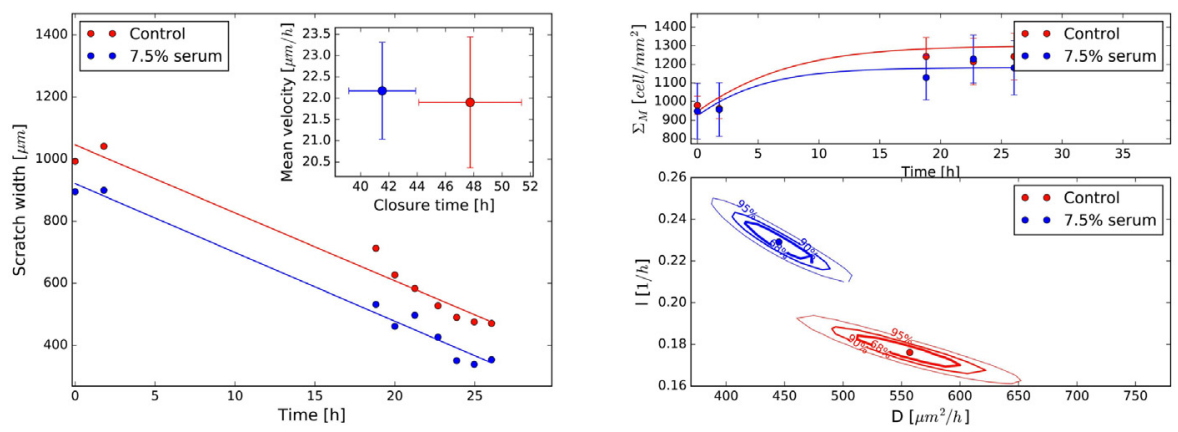

Fig. 9. Fit results for the $7.5 \%$ serum treat, experiment C. Same indications of the previous figure.
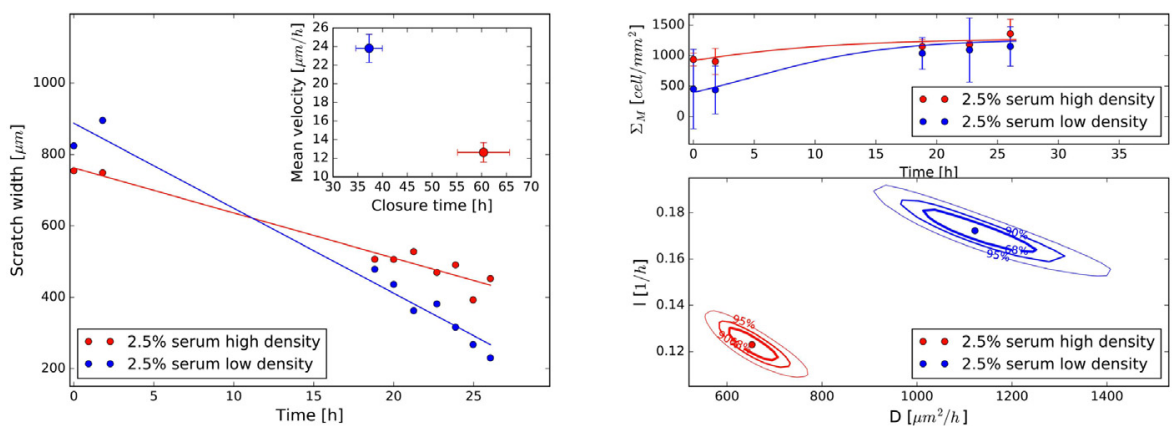

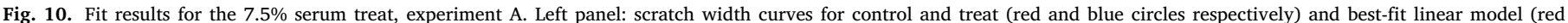

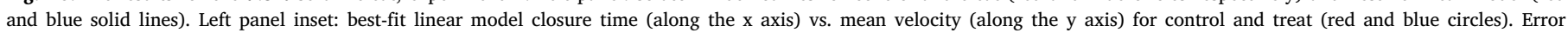

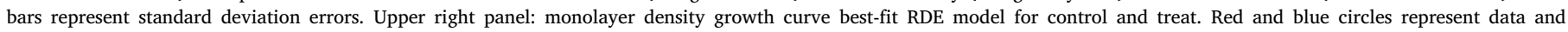

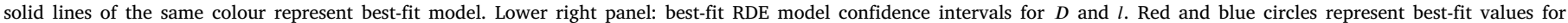
treat and control samples respectively ( $x$ coordinate represents $D$ and $y$ coordinate $l$ ). Contours of the same colour represent the relative confidence intervals.

controls) induces variations in the scratch evolution that the model estimates as not statistically significant and, in other words, that fall below the sensitivity limit of the method.

Another aspect to be observed is that 1 is the parameter with the worst intra-experiment reproducibility. As described above this parameter is constrained both by the fit of the density profiles $P^{m}(x, t)$ and by the preliminary fit of the monolayer growth curve $\Sigma_{M}\left(t^{\prime}\right)$. The limited number of points $t^{\prime}$ together with the night-time data acquisition gap can explain the worse reproducibility of the constraining of $l$. Of course, due to the observed $D-l$ correlation, we can expect that each results variation for one parameter reflect on the other (i.e. the lower is $l$ the higher will be $D$ and vice-versa).

The quality of the fit of $\Sigma_{M}\left(t^{\prime}\right)$ is also an important aspect when judging the quality of the whole fit. The experimental reproducibility of the initial monolayer density is an important parameter. In [20] the importance of initial conditions in model validation and interpretation for Reaction-Diffusion problems (in particular the starting monolayer condition in scratch assay) is pointed out. This aspect need certainly more testing in the future. In detail if two scratch assays are characterized by different initial monolayer density $\Sigma_{0}$ it is reasonable that they will show different scratch evolutions and the different results will be not due to the modelling procedure. Therefore it will be not correct to consider these samples statistical repetitions of the same experiment. The dedicated test presented in Section 3.3 confirms indeed this observation: halving the initial monolayer density while maintaining fixed the rest of the assay protocol, causes however a faster scratch closure interpreted by the RDE model as an increase of both the proliferation and the migration.

Moreover the final (i.e. limit) monolayer density $K$ of a cell culture made on a fixed support should be consistent in each experiment repetition. The measurement in two scratch assays of different $K$ values could be an indication that proliferation has been significantly different in the samples and thus, also in such a case, them cannot be correctly considered statistical repetitions of the same experiment.

\section{Conclusions}

This work, intended as the first of a series, had the aim of testing the modelling method with samples for which the effects of the treatments adopted are known and well characterized from a biological point of view: "known" samples are used to evaluate the "unknown" model.

The presented tests indicate that the method can distinguish samples and thus partially disentangle the contribution of migration and proliferation and we identified, in our particular set of experiments, a case $(7.5 \%$ serum culture medium) that constitutes a sensitivity limit of the method.

The good intra-experiment reproducibility, where for "good" we intend "with intra-experiment variations consistent with single-fit uncertainties" gives promising indications about the method robustness taken into account the discussed aspects regarding the quality of the fit and the fact that a better reproducibility has been obtained for treat-control parameter variations than for their absolute values.

We believe that an advantage of the proposed modelling is that it can be used with common microscopy data, not needing peculiar instrument setups and the principal effort residing in computer calculus time for RDE numerical solving. Of course a fundamental aspect is that the quality of a fit is principally determined by the ability to provide an adequate number of data points. In our experiments we adopted a $\sim 1 / \mathrm{h}$ sampling rate providing $\sim 15$ data points that resulted sufficient for constraining the model parameters as observed.

Once tested the method earned the confidence to be used to study unknown and interesting biological effects on the scratch assay: the "known" method, taking into account its uncertainties, bias and critical points, can be used to evaluate "unknown" samples. In future works we will adopt this technique to study aspects like the effect on migration and proliferation of physical stimuli known to influence wound healing processes (e.g. laser light or electromagnetic fields treatments) or of biological/biochemical factors and conditions (e.g. gradients or variation of concentration of nutrients, drugs, signalling molecules or specific growth factors). 


\section{Declaration of competing interest}

The authors declare that they have no known competing financial interests or personal relationships that could have appeared to influence the work reported in this paper.

\section{References}

[1] N. Pouliot, A. Burrows, Investigating Metastasis using in Vitro Platforms, Madame Curie Bioscience Database, Landes Bioscience, Austin (TX), 2000.

[2] C.A. Reinhart-King, Methods of Enzymology, Elsevier, Inc, 2008, pp. 45-61, (Chapter 3).

[3] C.C. Liang, A.Y. Park, J.L. Guan, In vitro scratch assay: a convenient and inexpensive method for analysis of cell migration in vitro, Nat. Protoc. 2 (2) (2007) 329-333.

[4] N.L. Kramer, A. Walzl, C. Unger, M. Rosner, G. Krupitza, M. Hengstschläger, H. Dolznig, In vitro cell migration and invasion assays, Mutat. Res. 752 (1) (2013) 10-24.

[5] A. Stamm, K. Reimers, A. Strauß, P. Vogt, T. Scheper, I. Pepelanova, In vitro wound healing assays state of the art, Bio. Nano Mat. 17 (1-2) (2016) 79-87.

[6] J.E. Jonkman, J.A. Cathcart, F. Xu, M.E. Bartolini, J.E. Amon, K.M. Stevens, P. Colarusso, An introduction to the wound healing assay using live-cell microscopy, Cell. Adh. Migr. 8 (5) (2014) 440-451, http://dx.doi.org/10.4161/cam.36224.

[7] M.N. Walter, K.T. Wright, H.R. Fuller, S. MacNeil, W.E. Johnson, Mesenchymal stem cell-conditioned medium accelerates skin wound healing: an in vitro study of fibroblast and keratinocyte scratch assays, Exp. Cell. Res. 316 (7) (2010) 1271-1281.

[8] G. Topman, O. Sharabani-Yosef, A. Gefen, A standardized objective method for continuously measuring the kinematics of cultures covering a mechanically damaged site, Med. Eng. Phys. 34 (2) (2012) 225-232.
[9] P. Masuzzo, M. Van Troys, C. Ampe, L. Martens, Taking aim at moving targets in computational cell migration, Trends Cell. Biol. 26 (2016) 88-110.

[10] P.K. Maini, S. McElwain, S. Leavesley, McElwain, I. David, Travelling waves in a wound healing assay, Appl. Math. Lett. 17 (6) (2004) 575-580.

[11] A.Q. Cai, K.A. Landman, B.D. Hughes, Multi-scale modeling of a wound-healing cell migration assay, J. Theoret. Biol. 245 (2007) 576-594.

[12] M.J. Simpson, K.A. Landman, B.D. Hughes, Cell invasion with proliferation mechanisms motivated by time-lapse data, Physica A 389 (18) (2010).

[13] S. Petrovskii, N. Shigesada, Some exact solutions of a generalized Fisher equation related to the problem of biological invasion, Math. Biosci. 172 (2) (2001) 73-94.

[14] S.T. Johnston, E.T. Shah, L.K. Chopin, D.L.S. McElwain, M.J. Simpson, Estimating cell diffusivity and cell proliferation rate by interpreting incucyte zoom assay data using the Fisher-Kolmogorov model, Syst. Biol. 9 (2015) 38.

[15] W. Jin, E.T. Shah, C.J. Penington, S.W. McCue, L.K. Chopin, M.J. Simpson, Reproducibility of scratch assays is affected by the initial degree of confluence: Experiments, modelling and model selection, J. Theor. Biol. 390 (2016) 136-145.

[16] S.W. McCue, W. Jin, T.J. Moroney, K. Lo, S. Chou, M.J. Simpson, Holeclosing model reveals exponents for nonlinear degenerate diffusivity functions in cell biology, Physica D 398 (2019) 130-140, http://dx.doi.org/10.1016/j.physd. 2019.06.005.

[17] K.W. Morton, D.F. Mayers, Numerical Solution of Partial Differential Equations: An Introduction, second ed., Cambridge, 2005, pp. 18-19, 24-25, 55.

[18] W. Jin, E.T. Shah, C.J. Penington, S.W. McCue, P.K. Maini, M.J. Simpson, Logistic proliferation of cells in scratch assays is delayed, Bull. Math. Biol. 79 (5) (2017) 1028-1050, http://dx.doi.org/10.1007/s11538-017-0267-4.

[19] Y. Avni, Energy spectra of X-ray clusters of galaxies, Astrophys. J. 210 (1976) 642-646.

[20] D. Warne, R.E. Baker, M.J. Simpson, Using experimental data and information criteria to guide model selection for reaction-diffusion problems in mathematical biology, Bull. Math. Biol. 81 (2019) 1760-1804. 